\title{
Meinung
}

Andreas Degkwitz*

\section{Der Kosmos eines dritten Ortes}

\author{
Gedanken zur Bibliothek von heute
}

https://doi.org/10.1515/bfp-2020-0018

Zusammenfassung: Im Zuge des digitalen Wandels sind Bibliotheken in der Situation, tiefgreifende Innovationsherausforderungen aufzugreifen und in ihren Serviceportfolios zu realisieren. Dabei reichen die Konzepte weit über die wechselseitigen Einflüsse analoger und digitaler Medien hinaus. Vielmehr geht es um neue Bibliotheksmodelle, wie die des „dritten Ortes“ oder der „Lernräume“. Dabei stellt sich die Frage, inwieweit sich Bibliotheken mit solchen Weiterentwicklungen tatsächlich neu erfinden oder ob sie Modelle aufgreifen, die in der Vergangenheit bereits praktiziert wurden. Aktuell spielt das Thema „Openness“ eine große Rolle für Bibliotheken, das mit „Open Access“ seinen Anfang nahm, inzwischen aber deutlich darüber hinausgeht. Mit Buchbeständen, die oft weit in die Vergangenheit zurückreichen, hat der „dritte Ort“ der Bibliothek aber auch etwas, das als gegenläufig $\mathrm{zu}$ unserer fortschrittsorientierten Zeit zu begreifen wie auch zu begrüßen ist.

Schlüsselwörter: Digitalisierung; dritter Ort; Innovation; Lernraum; Open Access; Openness

\section{The Cosmos of the Third Place Thoughts about the library of today}

Abstract: In the course of digital change libraries are in a position to take up profound innovation challenges and to implement them in their service portfolios. The concepts extend far beyond the mutual influences of analogue and digital media. The focus is rather on new library models such as the "third place" or "learning spaces". This raises the question of the extent to which libraries actually reinvent themselves with such further developments or whether they take up models that have already been practised in the past. At the moment, the topic of

1 The online version of this article offers an English version of the text as supplementary material (https://doi.org/10.1515/bfp-2020-0018).

*Kontaktperson: Andreas Degkwitz, andreas.degkwitz@ub.hu-berlin.de “openness” plays a major role for libraries, which began with 'Open Access' but has since gone much further. With book holdings often dating far back into the past, the "third place" of the library also has something to be understood and welcomed as contrary to our progressoriented times. ${ }^{1}$

Keywords: Digitisation; third place; innovation; learning space; open access; openness

Mit der Digitalisierung der Medien wurde die Traditionseinrichtung „Bibliothek“ an die Spitze der Innovation katapultiert. Wer hätte geglaubt, dass dieser Hort menschlicher Wissensgüter mit seinen vielen Formaten und unterschiedlichen Formen an Handschriften und Druckwerken im digitalen Zeitalter einen Platz in der ersten Reihe findet? Es gibt immer noch viel zu viele, die das nicht für möglich halten und in Bibliotheken entweder ihre Kinder- und Jugendzeit wieder erleben oder mit Bibliotheken anachronistische, weltfremde Bildungsromantik verbinden. Denn hatten Bibliotheken bisher über Jahrhunderte papiergebundene Bücher und Zeitschriften dauerhaft für Nutzungen zur Verfügung gestellt, tauchten um die Jahrtausendwende die ersten E-Journals und E-Books auf. Da hatten WEB-Server schon Eingang in die Bibliotheken gefunden und sind schnell zur Standardinnovation geworden. Mit dem WEB-Server war der neue Zugang, das Gateway, die Plattform, das Portal der digitalen Bibliothek geschaffen, die zunächst Katalog, Öffnungszeiten und „Über uns“ präsentierte.

Allerdings waren die Bibliotheken mit diesem Modus des Zugangs nicht mehr allein und die einzigen, die Literatur und Informationen verbreiteten, so dass sie den „Zugang zum Wissen“ nicht mehr als „Monopol“ für sich beanspruchen konnten - da waren sie mit ihren gedruckten Beständen besser dran. Denn wer wollte schon diese Massen an Büchern erwerben, erschließen, zur Verfügung stellen und auf Dauer magazinieren, um sie „nur“ wieder und wieder ausleihen zu können und um sich insgesamt seitenweise verstaubten Papiers anzunehmen? Im Gutenberg-Zeitalter war genau dieses heroische Back-Office das Privileg dieser magazinreichen Burgen des Menschheits- 
gedächtnisses wie der Schatzkammern abendländischen Wissens. Doch mit dem Internet standen Bibliotheken ganz plötzlich und von Anfang an im Wettbewerb mit Informationsanbietern, die ebenfalls über das WWW Informationen verfügbar machten und, waren dies Firmen oder privat-wirtschaftlich agierende Unternehmen, wurden auf diesem Wege sogar Informationen verkauft. Zum Leidwesen der Bibliothekare verstanden es die kommerziellen Wettbewerber meistens besser als sie, ihre Inhalte wirkungsvoll zu vermarkten und ihren Kunden nahe zu bringen. Heiter bis wolkige Orakel damaliger Influencer oder Technologieexperten ließen verlauten, dass Bibliotheken kurz vor dem Ende ihres Fortlebens stünden, komplett vom Internet abgelöst und von diesem ersetzt werden würden. Vieles werde man brauchen, doch Bibliotheken gehörten definitiv zu einem obsolet werdenden Zweig traditioneller Bildungsinstitutionen, die den technologischen Wandel hin zur Beglückung der Menschheit ebenso wenig überleben würden wie das „Buch“ als ihr corpus delicti: Beides sei ans Ende seiner Laufzeit gekommen, habe sein Verfallsdatum erreicht und werde nun in der Informationsflut verschwinden, auf der nur das WWW als Arche Noah schwimmen könne.

Im Kontext solcher Prognosen wurde gern ignoriert, dass sich Bibliotheken zum einen weiterhin vieler Besuche erfreuten. Zum anderen wurden seit der Jahrtausendwende viele neue Bibliotheken gebaut oder renoviert. Angesichts der eifernden Untergangsprognosen hätte vor allem letzteres in Staunen versetzen können: Bibliotheken doch noch nicht obsolet? Aktuell sogar mehr geliebt als zuvor? Ein Fall von „Willkommen und Abschied“? Was da geschah, war in der Tat überraschend. Denn dass auch Bibliothekare in der Lage waren, ihre Bibliotheken im digitalen Wandel neu zu erfinden, hätte wohl kaum jemand für möglich gehalten. Offenbar gab es noch Argumente für Bibliotheken jenseits von Desktop und Internet. Die Planungskonzepte für neue Bibliotheksgebäude gaben dafür sichtbaren Anlass. Neue Visionen und Zielsetzungen für Bibliotheken wurden aber auch unabhängig von Bauprojekten entwickelt.

Eine beachtliche Vielfalt von Weiterentwicklungsoptionen hatte sich bald herausgestellt und wurde intensiv diskutiert: Bibliotheken der Zukunft waren digital, elektronisch, hybrid und hypertextuell, Wissenscontainer, Informationshosts, Peer-to-Peer-Netzwerke, dritte Orte und Lernräume, virtuelle Forschungs- und Lernumgebungen was künftig alles „Bibliothek“ sein könnte und was „Bibliothek“ überhaupt sei, war eine nicht versiegende Quelle von Inspiration, Innovation und mal mehr, mal weniger wissensbasiertem Streit. Allen Bemühungen um die Neuausrichtung von Bibliotheken gemeinsam war die Auffas- sung, dass der gedruckte Bestand, der meistens als analog oder physisch bezeichnet wurde, nicht mehr im Zentrum des bibliothekarischen Fokus stehen, vielmehr seinen Platz an die digitalen Ressourcen abgeben sollte, die diesen budgetmäßig schon besetzten. Diese Verlagerung beförderte die Vision wie die Befürchtung, dass Druckwerke gänzlich von digitalen Medien ersetzt werden würden und in naher Zukunft wohl gar keine Rolle mehr spielten. In dieser Weise passte man sich entweder ablehnend oder befürwortend fortschreitender Infragestellung von Bibliotheken an.

Die Entwicklung des Buchmarktes hat die Infragestellung von Bibliotheken nicht bestätigt noch gaben die Lese- und Rezeptionspräferenzen der Nutzer zu erkennen, dass Bücher für sie bedeutungslos würden. Vielmehr vermittelte sich der Eindruck, dass sich die digitalen Versionen von Büchern und Zeitschriften stärker an ihren gedruckten Ahnen als an den Kindern der digitalen Revolution orientierten. Dauerthema war vor diesem Hintergrund der permanent überstrapazierte, angeblich unüberbrückbare Gegensatz zwischen „Analog“ und „Digital“. Dieser Gegensatz wurde behauptet, aber im Regelfall nicht erklärt und hat im Ergebnis meistens zu dem Befund schlichter Medienvielfalt geführt. Denn anstelle sich unversöhnlich gegenüberzustehen, ergänzen sich „Analog“ und „Digital“ - diese Einsicht fuhr einen Konflikt zurück, der oftmals aufgebauscht wurde, ohne dies begründen zu können. Von daher ist viel interessanter, was der oft adressierte „dritte Ort“, den Bibliotheken abgeben möchten, wirklich an Innovation bescherte und bot. Nein, "dritte Orte“ sind keine Avatare oder Locations in „Second life“, wo Bibliotheken gegen Zahlung von LindenDollars Grundstücke für virtuelle Präsenzen kauften - erinnert sich daran noch jemand?

Der „dritte Ort“ ist ein Terminus aus der Sozialwissenschaft, der „public locations“ beschreibt, die neben dem „ersten Ort“ der Wohnung und dem „zweiten Ort“ des Arbeitsplatzes an dritter Stelle der Lebensentfaltung stehen und Menschen in bewegten oder gestalteten, öffentlich zugänglichen Räumen das Lebensglück von Austausch, Begegnung und Kommunikation sowie von Information, Vergnügen und Zerstreuung bieten und oftmals „wohnzimmerartig“ ermöglichen. „Dritte Orte“ sind öffentliche Orte wie Bahnhöfe, Buchhandlungen, Department-Stores, Einkaufszentren, Hotel-Lobbys, Konzerthallen, Museen, Opernhäuser, Restaurants, Theater, Shopping-Malls und eben auch Bibliotheken. Dabei sind Bibliotheken nicht einfach die besseren Internet-Hotspots mit möbel- und raumseitig hoher Aufenthaltsqualität, sondern Orte der Bildung, Gesellschaft, Information, Kreativität, Kritikfähigkeit und Kompetenzentwicklung, wie es die Nutzer sich wünschen. 
Die Bibliothek als „dritter Ort“ ist ein Lebensraum, der für Weiterentwicklung und Wohlbefinden seiner Nutzer viel tut, der aber Literatur- und Informationsversorgung, die eine Bibliothek auch als „dritter Ort“ durchaus leistet, eher als ein Angebot unter vielen sieht. Die Nutzer stehen als Nachfrager im Mittelpunkt des Geschehens, nicht mehr, wie oftmals in den Jahrhunderten zuvor nachgesagt, die Objekte, die Bibliotheken schon von ihrer Bezeichnung her charakterisieren, nämlich schlicht und ergreifend die Bücher.

Diese betriebswirtschaftliche Logik begleitete Bibliotheken als „dritte Orte“ auf dem Weg ihrer Neuerfindung, wie sie sich auch auf Lernräume und Makerspaces bezog davon später noch mehr. Dabei drängt sich die Frage auf, ob „dritte Orte“ eine Erfindung des 21. Jahrhunderts waren oder ob es solche Orte - auch als Bibliotheken - nicht schon zu früheren Zeiten gab. Den „öffentlichen Ort“ hat es immer gegeben und seine Tradition reicht wie die von Bibliotheken weit zurück. „Öffentlich“ ist im Allgemeinen auch ,jeder und jedem zugänglich“. Doch wem Bildung, Kapital oder Zeit fehlen, wird sich nicht an Orten aufhalten wollen, für deren Besuch genau dies die Voraussetzung ist. So waren auch Bibliotheken stets „dritte Orte“ derer, die in der Lage waren, Bibliotheken tatsächlich zu nutzen und sich auf den Umgang mit Bibliotheken verstanden, um sich dort auszutauschen, zu informieren, zu kommunizieren, kreativ zu sein und zu zerstreuen. Mit dem „dritten Ort“ entdecken wir deshalb eher etwas wieder, was Bibliotheken immer charakterisierte, als dass wir Bibliotheken tatsächlich neu erfinden. Vielleicht ist das überhaupt die List der Neuerfindung von Traditionen, dass „ein guter, alter Wein auch in neuen Schläuchen“ an Bekömmlichkeit nicht verliert.

Nicht weniger interessant als der „dritte Ort“ sind die „Lernräume“, als welche sich Bibliotheken gerne ebenfalls neu profilieren. Denn lernen, ja sogar lebenslang lernen, müssen wir alle - dies selbstverständlich in der Informationsgesellschaft, die wir zunächst erlebt hatten, und nun in der Wissensgesellschaft, in der wir uns heute deshalb so wohl fühlen, weil wir uns darin als Wissensträger verstehen, ohne die die Wissensgesellschaft nicht existieren kann. Dies gilt sowohl für die Bibliothekare als auch für die Nutzer von Bibliotheken. Wer über Wissen verfügen möchte, ist in der Situation, sich Wissen anzueignen, also zu lernen - dafür bieten Bibliotheken als Horte und Vermittler von Wissensgütern beste Voraussetzungen. Der „Lernraum Bibliothek“ könnte sich also als „Kaufhaus des Wissens“ verstehen, als „KdW“ derjenigen, die zu lernen bereit und - mehr noch - dazu verpflichtet sind. Unausgesprochen ist sich die Wissensgesellschaft darin einig, deutlich mehr zu wissen als jede Gesellschaft zuvor. Wis- sen vermehrt sich in unseren Zeiten exponentiell, wie auch die ebenso steigende Anzahl wissenschaftlicher Publikationen zeigt. Eher im Ausnahmefall hingegen wirft die Wissensgesellschaft die Frage auf, ob aufgrund ihrer enormen Wissensentwicklung die Individuen der Wissensgesellschaft mehr wissen als die Individuen aller Gesellschaften vor der Wissensgesellschaft. Anders gefragt: Werden wir alle dem Anspruch unserer Wissensgesellschaft gerecht?

Die beste Antwort auf diese Frage ist: „Nein, ich entspreche dem Anspruch der Wissensgesellschaft nicht und werde ihm nicht entsprechen können. Doch meine Bibliothek ermöglicht mir den Zugang zu den Ressourcen, die ich benötige, um in der Wissensgesellschaft wirklich bestehen zu können." Jetzt sage noch jemand, dass Bibliotheken über keine Systemrelevanz verfügen. „Googeln“ solche noch, die dies leugnen, oder „Bibliotheken“ die schon? Tja, Google - Google fordert schmerzhaft heraus, trifft Bibliotheken bisweilen ins Herz und auf jeden Fall an der Achillesferse: Hier der Suchschlitz, der den Rohstoff „Information“ zutage fördert - dort das Discovery-System oder der Katalog, der OPAC heißt, um den Nutzern Suchergebnisse auf dem Silbertablett zu servieren: Noch Fragen, wer das Sagen in „Lernräumen“ hat und welche Institution alle, die in unserer Wissensgesellschaft suchen und finden, substanziell unterstützt?

Doch wie beim „dritten Ort“ wirft bei den „Lernräumen" auch die List der Neuerfindung Bibliotheken auf sich zurück. Denn findet „Lernen“ in Bibliotheken erst in der Wissensgesellschaft statt oder erfolgte dies auch schon zuvor? Haben Menschen schon immer in Bibliotheken gelernt oder machen sie das erst jetzt? Ist „Lernen“ die einzige Destination von Bibliotheken und „Forschen“ bleibt außen vor? Die alten Griechen ließen sich auf den Schultern von Wissensriesen tragen und waren so kühn, über die Meere in alle Welt zu tragen, was sie auf den Schultern der Riesen erfuhren - so weiß es Herodot. Ich weiß es nicht anders und weiß mit den alten Griechen, dass damit auch die Voraussetzungen für Forschung gegeben waren und sind. In der Tradition der Humboldt-Brüder ist die Einheit von Lehre und Forschung Konsens - das machen Bibliotheken möglich und haben sie immer möglich gemacht. Sind „Lernräume“ wirklich Innovationen, die sich seit Kurzem für Bibliotheken regelrecht auftun?

Dass die nun neu erfundenen Bibliotheken fortbestehen und hohe Wertschätzung haben, erfreut. Dass die neu erfundenen Bibliotheken gegenüber früheren Bibliotheken nicht über ein grundsätzlich neues Selbstverständnis verfügen, ist nicht untypisch für Innovation. Denn Innovation erfolgt meistens organisatorisch oder technisch und verändert den Status quo, doch orientiert sie sich im Regelfall 
weiterhin an der Definition der Strukturen, die der Erneuerung unterliegen. Anders gesagt, verändern sich Bibliotheken nicht im Kern, doch greifen sie - wie auch bisher organisatorische und technische Innovationen zur Verbesserung ihrer Services auf. Bisher ist das immer gelungen. Ob dies im Zuge des digitalen Wandels gelingt, ist sehr wahrscheinlich, doch nicht immer ganz evident. Die Verunsicherung, die daraus resultiert, führt zu Infragestellung und Zweifel, der sich aufs Grundsätzliche überträgt, ohne dass dafür wirklich Anlass besteht. Denn Markenzeichen, mit dem Bibliotheken den technischen Wandel aufgreifen und realisieren, ist nicht technisch, sondern politisch und heißt „Open“. So einfach und selbstverständlich dieser Claim auch ist, impliziert er doch zahlreiche Fragen, gibt sich aber auf jeden Fall innovativ.

„Open“ (nicht „Offen“) gründet auf „Open Access“ nein, nicht „Offener Zugang“, sondern „Open Access“. Warum ist das so wichtig? Mit „Open Access“verändert die digitale Transformation das Publikationsmodell seit gut zwanzig Jahren. Anstatt Publikationen als Monografien oder Zeitschriften(artikel) zu kaufen oder in anderer Weise von Buchhandlungen oder Verlagen für den Bestandsaufbau oder die Sammlungen der Bibliotheken zu beziehen, konnten mit geeigneter Technik elektronische Publikationen von den Autoren selbst (und ohne Unterstützung von Verlagen) hergestellt und über das Internet weltweit verbreitet werden. Das Lesen und Rezipieren von OpenAccess-Veröffentlichungen kostet für Leser und Nutzer von Bibliotheken nichts. Was folgte daraus für Bibliotheken? Mit dieser Möglichkeit des Selfpublishing wurden Bestandsaufbau und Sammlungsauftrag perspektivisch durchaus infrage gestellt - zu einer unmittelbaren Infragestellung kam es deshalb allerdings nicht. Doch auf jeden Fall wurde aus dieser Einsicht die Konsequenz gezogen, dass auch Bibliotheken in der Lage sein sollten, Publikationsleistungen zu erbringen.

Seither gründeten die meisten wissenschaftlichen Bibliotheken Deutschlands Universitätsverlage und betreiben diese bis heute. Zugleich bemühte man sich bei Verlagen um die Freigabe der Zweitveröffentlichung (GreenRoad-Open-Access) und um die Realisierung primären „Golden-Road-Open-Access“. Neben „gold“ und „grün“ kam es noch zu weiteren Farben oder Edelmetallen, um weitere Open-Access-Modelle zu charakterisieren, die neben „grün“ oder „gold“ florierten. So wurden im Zuge des sich wandelnden Publikationsmodells in Bibliotheken neue Aufgaben geschaffen. Mit dem Eigenverlag war der Preiswettbewerb für den subsidiären Publikationsbereich auf jeden Fall zu gewinnen. Dass davon auch der primäre Publikationsbereich nicht unberührt blieb, sei hier nicht ausgeführt, aber natürlich erwähnt. Bis heute wird über
Open Access viel mit Verlagen kooperiert. Zusätzlich wurde die Aufgabe „Open Access“ dadurch legitimiert, dass Publikationen auch informationstechnisch aufbereitet und verarbeitet werden mussten. Inzwischen haben sich umfassende Portfolios an Infrastrukturen, Services und Werkzeugen etabliert, die den damit von Bibliotheken beanspruchten Innovationsbereich unmittelbar zu erkennen geben und deutlich positionieren, dass es ihnen um „Open“ geht. In der Tat macht Open-Access-Publizieren viel Sinn - auch über Bibliotheken hinaus.

„Open“ wurde zum Mantra der Weiterentwicklung von Bibliotheken. „Open Library“ weckte den Eindruck, als werde Offenheit für Bibliotheken jetzt erst neu entdeckt und sei zudem als Erfolg des digitalen Wandels zu sehen. Was tatsächlich offen bleibt, ist das Verständnis von „Open“ selbst und was „Openness“ eigentlich anvisiert. Denn Offenheit und Zugänglichkeit haben Bibliotheken eigentlich immer geprägt. Wo läge sonst auch ihr Sinn? Nicht auszuschließen ist dabei, dass es eingeschränkt zugängliche Bibliotheken teilweise gab und noch gibt. Doch der Regelfall ist das nicht - jedenfalls nicht in Ländern und Regionen, in denen die Freiheit der Information und der Rede gilt. Vor diesem Hintergrund stellt sich umso mehr die Frage, was „Open“ eigentlich meint.

Der Schlüssel zur Antwort auf diese Frage mag in den Möglichkeiten liegen, digitale Arbeitsformen denjenigen zu bieten, die über Bilder, Daten und Texte interaktiv und vernetzt zusammenarbeiten wollen. Voraussetzung dafür sind Standards, die kooperatives Arbeiten technisch erfordert. Standards sind aber auch notwendig, um Forschungsergebnisse nachzunutzen und nachzuvollziehen. Von daher gelten sie für Publikationen wie auch für Forschungsdaten, Methoden und Softwareapplikationen. Nicht zuletzt gelten Standards auch für den Nachweis, um digitale Publikationen oder Objekte dauerhaft wiederzufinden. Angesichts dieser Anforderungen hat „Openness" einen hohen Stellenwert, aber auch den Preis der Standardisierung, die allein im Anspruch digitaler Verarbeitbarkeit von Bildern, Daten und Texten begründet ist.

Es wäre ganz falsch zu behaupten, dass uns der digitale Wandel zum ersten Mal Standards lehrt. Standards sind uns selbstverständlich auch unabhängig von digitalen Arbeits- und Lebensformen vertraut. Doch dass Standards unsere Arbeits- und Lebensabläufe so sehr beeinflussen, wie es uns die Digitalisierung erleben lässt, und sie uns deshalb gleichsam beherrschen, ist in der Tat ein Novum. Wir erfahren jetzt in der durch Corona entstandenen Krise, dass allein und einzig Informationstechnik Austausch, Interaktion und gemeinsame Arbeit ermöglicht. Doch die Aussicht, sich darauf beschränken zu müssen, wird rasch als Verlust empfunden. Berechtigt ist deshalb die Frage, 
ob wir im Kontext von Open-Access-Standards oder Inhalte priorisieren. Auf was kommt es vorrangig an - auf die inhaltliche Qualität von Veröffentlichungen oder auf Standards zu deren Erstellung und Distribution? Denn mögen Daten und Publikationen „Open“ sein. Ist „Open“ Leitkriterium für die Bewertung von Publikationen, wird Qualität von Inhalten schnell auf der Strecke bleiben und künftig nachrangig sein. Das will wirklich niemand - auch „Open Libraries" nicht.

Ein Beitrag, der Bibliotheken als erfolgreiches Beispiel für „Dauer im Wechsel“ beschreibt, könnte dazu verleiten, in Bibliotheken das Wahre, das Schöne und vor allem das Gute zu sehen als ein Relikt vergangener Zeiten, das sich immer aufs Neue im Jetzt wie auch künftig regeneriert. Ohne Zweifel bereichert die Bewegung, in der sich Bibliotheken dabei befinden, Bibliothekare und ist zugleich für Nutzer von Bibliotheken von Vorteil. Allerdings geht diese Bewegung verloren, wenn die Emphase für Bibliotheken zum Denkmal wird. Denn um ein Denkmal zu sein, stehen Bibliotheken zu sehr im Leben. Darüber hinaus haben Bibliotheken etwas, das dem Zeitgeist zuwider läuft, etwas von Widerspenstigkeit, die vielleicht überrascht. Denn sie konfrontieren die Gegenwart, die morgen zum Gestern geworden ist, mit Vergangenem, das wir in Bibliotheken suchen, finden und rezipieren, um uns auf diese Weise Künftiges zu erklären. Anders gesagt, führt uns der bibliothekarische Kosmos einerseits weiter nach vorne wie er uns andererseits wieder zurückführt - das ist etwas, das sich dem hektischen Aufwärtsstreben unserer fortschrittsverwöhnten Dezennien widersetzt, durchaus befreiend wirkt und außerordentlich inspiriert. Deshalb schätzen wir diese „dritten Orte“ und „Bibliotheken“ tatsächlich lieber statt nur zu „googlen“.

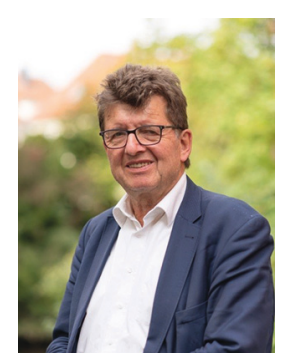

Andreas Degkwitz

Universitätsbibliothek der Humboldt-

Universität zu Berlin

Unter den Linden 6

D-10099 Berlin

andreas.degkwitz@ub.hu-berlin.de 